\title{
Thin Circular Diamond Membrane with Embedded Nitrogen-Vacancy Centers for Hybrid Spin-Mechanical Quantum Systems
}

\author{
S. Ali Momenzadeh, ${ }^{1, *}$ Felipe Fávaro de Oliveira, ${ }^{1}$ Philipp Neumann,,${ }^{1}$ D. D. Bhaktavatsala Rao, ${ }^{1,2}$ Andrej Denisenko, ${ }^{1}$ \\ Morteza Amjadi, ${ }^{3}$ Zhiqin Chu, ${ }^{1}$ Sen Yang, ${ }^{1}$ Neil B. Manson, ${ }^{4}$ Marcus W. Doherty, ${ }^{4, \dagger}$ and Jörg Wrachtrup ${ }^{1,2}$ \\ ${ }^{1} 3 r d$ Institute of Physics, Research Center SCoPE and IQST, University of Stuttgart, \\ 70569 Stuttgart, Germany \\ ${ }^{2}$ Max Planck Institute for Solid State Research, Heisenbergstrasse 1, 70569 Stuttgart, Germany \\ ${ }^{3}$ Physical Intelligence Department, Max Planck Institute for Intelligent Systems, \\ Heisenbergstrasse 3, 70569 Stuttgart, Germany \\ ${ }^{4}$ Laser Physics Centre, Research School of Physics and Engineering, Australian National University, \\ Australian Capital Territory 0200, Australia \\ (Received 2 May 2016; revised manuscript received 2 August 2016; published 31 August 2016)
}

\begin{abstract}
Coupling mechanical degrees of freedom to single well-controlled quantum systems has become subject to intense research recently. Here, we report on the design, fabrication, and characterization of a diamond architecture consisting of a high-quality thin circular diamond membrane with embedded near-surface nitrogen-vacancy centers (NVCs). To demonstrate this architecture, we employ the NVCs by means of their optical and spin interfaces as nanosensors of the motion of the membrane under static pressure and in-resonance vibration. We also monitor the static residual stress within the membrane using the same method. Driving the membrane at its fundamental resonance mode, we observe coupling of this vibrational mode to the spin of the NVCs. Our realization of this architecture can manifest the applications of diamond structures in 3D piezometry such as mechanobiology and vibrometry, as well as mechanically mediated spin-spin coupling in quantum-information science.
\end{abstract}

DOI: 10.1103/PhysRevApplied.6.024026

\section{INTRODUCTION}

Quantum hybrid systems (QHSs) consisting of quantum objects and high-quality mechanical structures have attracted significant research attention in recent years $[1,2]$. As a prominent qubit in a host with outstanding mechanical properties, negatively charged nitrogenvacancy centers (NVCs) have been considered in several proposals for applications in quantum-information science and nanoforce sensing [3-5]. NVCs in diamond possess excellent spin and optical properties [6] which deliver them distinguished capabilities for quantum technologies, such as high-sensitivity quantum nanosensing of magnetic [7-9] and electric fields [10]. Complementing this fact, single-crystal diamond (SCD) features notable mechanical properties, like high Young's modulus and low intrinsic dissipation [11]. Combining these advantages, breakthrough steps have been taken by coupling the fundamental vibrational mode of diamond micro- $[12,13]$ and nanocantilevers [14] to the ground-state spin of NVCs [15-17].

Besides micro- and nanocantilevers, thin circular membranes are also well-known mechanical structures, being utilized in a wide range of applications, from high-precision pressure sensing [18] to observation of quantum effects such as cavity quantum electrodynamics (QED) [19]. For

\footnotetext{
*a.momenzadeh@physik.uni-stuttgart.de

† marcus.doherty@anu.edu.au
}

instance, silicon nitride $\left(\mathrm{Si}_{3} \mathrm{~N}_{4}\right)$ thin membranes with lateral dimensions of a few millimeters and submicron thickness have been extensively employed in QED experiments [20], e.g., in membrane-in-the-middle geometry [21]. Nevertheless, for some materials, like SCD, realization of such a prevalent structure [22] free from cracks and trenches remains a significant challenge. Furthermore, SCD-NVC hybrid systems show additional striking characteristics, like low cytotoxicity and long-term stability [24], which make them attractive for other applications [25]. For example, they could be designed for noninvasive, three-dimensional, and real-time measurement of extracellular forces (e.g., in traction force microscopy [26]). Towards such techniques, more detailed information about the mechanical properties of the diamond microand nanostructures, as well as the mechanical interaction of the NVCs, is required.

In this paper, we present a QHS based on NVCs embedded approximately $15 \mathrm{~nm}$ under the surface of a thin circular SCD membrane (hereafter referred to as "membrane"). This SCD membrane has a diameter of approximately $1.1 \mathrm{~mm}$, a thickness of approximately $1.2 \mu \mathrm{m}$, and a surface roughness of approximately $0.4 \mathrm{~nm}$, thus showing a diamater-to-thickness ratio of approximately 1000 . To examine the mechanical properties of this membrane, we employ NVCs as nanosensors coupled to different mechanical degrees of freedom of the membrane. We measure the fluorescence point-spread 
function (PSF) [27] of individual embedded NVCs by means of confocal microscopy to detect the deflection of the membrane under a static (dc) applied pressure. As a result, by applying the continuum mechanics theory (CMT) of thin membranes [18], we infer an effective thickness of approximately $1.2 \mu \mathrm{m}$ and an average radial residual stress of $54 \mathrm{MPa}$. Furthermore, by means of coupling of the ground-state spin levels of the NVCs to the mechanics of the membrane, we monitor the membrane under applied dc pressure and in-resonance vibration (ac), as well as its residual stress. In such a manner, we observe an approximately $2.3-\mathrm{MHz}$ spin-resonance frequency shift under 1 bar of applied dc pressure. In addition, a radial residual stress of approximately $52 \mathrm{MPa}$ is detected by means of the spins of single NVCs, which shows good consistency with the value derived from the confocal microscopy measurements and mechanics model of the membrane. Moreover, we perform further benchmark measurements, namely, the Hahn echo sequence, to acquire more detailed information from the spin-phonon coupling regime.

\section{CONTINUUM MECHANICS AND SPIN-STRESS MODEL}

Based on their thickness $(t)$ and central deflection $(w)$ under a given applied pressure $(P)$, circular membranes in CMT are classified into two main categories of thin and thick membranes [18]. In this work, we focus on developing thin SCD circular membranes since, according to CMT, thin membranes $(w>t)$ show a higher sensitivity to the applied pressure than thick ones $(w<t)$ [18]. The $P-w$ relation for thin circular membranes, neglecting bending moments, is given by

$$
P=\frac{4 t w}{R^{2}}\left(\sigma_{0}+\frac{2}{3} \frac{w^{2} E}{R^{2}\left(1-\nu^{2}\right)}\right),
$$

in which $\sigma_{0}$ and $R$ are the radial residual stress and the radius of the membrane, and $E \approx 1.2 \times 10^{12} \mathrm{~Pa}$ and $\nu=$ 0.069 are the diamond Young's modulus and Poisson's ratio, respectively. Hereafter, we assume that $1-\nu^{2} \approx 1$ in Eq. (1). As can be seen in Eq. (1), a thin membrane is expected to exhibit a nonlinear response to the applied pressure. In addition, for a given applied pressure, membranes with larger radii deflect more. Consequently, notwithstanding nonlinear behavior, maximum pressure sensitivity is achieved for the maximum possible radius and the minimum possible thickness.

Deflection of the diamond membrane under the applied pressure creates crystal stress in the diamond lattice, which alters the ground-state spin-resonance frequencies of the embedded NVCs through longitudinal and transverse shifts $[15,16,28-30]$. In this work, we are limited to only the longitudinal frequency shift, and thus the stress along the axis of the NVCs. In the cylindrical coordinate system $(r, \theta)$, the radial and tangential strain vs the central deflection for a thin circular diamond membrane can be written as [18]

$$
\epsilon_{R R}=\frac{2}{3} \frac{w^{2}}{R^{2}}, \quad \epsilon_{T T}=\frac{2}{3} \frac{w^{2}}{R^{2}}\left(1-\frac{r^{2}}{R^{2}}\right),
$$

respectively. To convert the central deflection of the membrane to the longitudinal shift in the NVC groundstate spin-resonance levels, we apply the spin-stress model [5]. In this model, the longitudinal frequency shift is expressed vs six different stress components of the stress tensor [31]:

$\Delta=\frac{1}{2}\left[A_{1}\left(\sigma_{x x}+\sigma_{y y}+\sigma_{z z}\right)+2 A_{2}\left(\sigma_{x y}+\sigma_{x z}+\sigma_{y z}\right)\right]$,

where $A_{1}$ and $A_{2}$ are constants equal 4.86 and $-1.55 \mathrm{MHz} / \mathrm{GPa}$, respectively [5]. For a (100) diamond surface, the matrix form of the stress tensors of each NVC orientation $(A-D)$ are given by

$$
\begin{aligned}
S_{A}=\left(\begin{array}{ccc}
\sigma_{x x} & \sigma_{x y} & 0 \\
\sigma_{x y} & \sigma_{y y} & 0 \\
0 & 0 & 0
\end{array}\right) & S_{B}=\left(\begin{array}{ccc}
\sigma_{x x} & 0 & -\sigma_{x y} \\
0 & 0 & 0 \\
-\sigma_{x y} & 0 & \sigma_{y y}
\end{array}\right) \\
S_{C}=\left(\begin{array}{ccc}
0 & 0 & 0 \\
0 & \sigma_{y y} & \sigma_{x y} \\
0 & \sigma_{x y} & \sigma_{x x}
\end{array}\right) & S_{D}=\left(\begin{array}{ccc}
\sigma_{y y} & -\sigma_{x y} & 0 \\
-\sigma_{x y} & \sigma_{x x} & 0 \\
0 & 0 & 0
\end{array}\right) .
\end{aligned}
$$

Therefore, the longitudinal frequency shifts corresponding to each orientation $(A-D)$ are as follows:

$$
\begin{aligned}
& \Delta_{A}=\Delta_{C}=\frac{1}{2}\left[A_{1}\left(\sigma_{x x}+\sigma_{y y}\right)+2 A_{2}\left(\sigma_{x y}\right)\right], \\
& \Delta_{B}=\Delta_{D}=\frac{1}{2}\left[A_{1}\left(\sigma_{x x}+\sigma_{y y}\right)+2 A_{2}\left(-\sigma_{x y}\right)\right],
\end{aligned}
$$

among which the different NVC orientations fall into two sets of 2, with distinct frequency-shift distributions $\left(\Delta_{+} \equiv \Delta_{A, C}\right.$ and $\left.\Delta_{-} \equiv \Delta_{B, D}\right)$. By converting the strain components from the cylindrical coordinate system to the Cartesian system [Eq. (2); details are addressed in Appendix A], we can rewrite Eq. (5) vs the central deflection $(w)$ for different positions of the membrane in the cylindrical coordinate system:

$\Delta_{ \pm}=\frac{1}{2} E\left[A_{1}\left(\frac{2 w^{2}}{3 R^{2}}\right)\left(2-\frac{r^{2}}{R^{2}}\right) \pm A_{2} \sin (2 \theta)\left(\frac{2 w^{2} r^{2}}{3 R^{4}}\right)\right]$.

\section{MEMBRANE-NVC DEVICE FABRICATION}

Our approach to fabricating such a hybrid device is as follows: prior to the membrane fabrication, NVCs are 
generated with the aid of a nitrogen implantation technique [32] with the energy and dose of $10 \mathrm{keV}$ and $50 \mu \mathrm{m}^{-2}$, respectively, in an approximately $(2 \times 2 \times 0.027)-\mathrm{mm}$ [100]-oriented single-crystal CVD electronic grade $\left({ }^{13} \mathrm{C}\right.$ natural abundance) diamond film [23]. The chosen implantation dose and energy result in single NVCs with an average depth of approximately $15 \mathrm{~nm}$ under the diamond surface, resolvable by confocal microscopy. Then, by means of an etching technique based on an $\mathrm{Ar} / \mathrm{SF}_{6}$ plasma-gas mixture and an auxiliary diamond with an angled-wall hole to act as an etching mask [Fig. 1(a)], the membrane is created from the SCD film by etching for a depth of $>25 \mu \mathrm{m}$ [Fig. 1(b)]. A crucial role is played by the angled wall of the hole in the diamond etching mask, which results in homogeneous etching with minor deviations over the whole membrane area (approximately $0.1 \%$ ). This mask also avoids the cracks and trenches at the membrane edge which appear in earlier attempts. Notably, the presented etching recipe achieves both a relatively high etching rate and a remarkably smooth final surface. The etching rate is measured to be approximately $170 \mathrm{~nm} / \mathrm{min}$, followed by a surface roughness of approximately $0.4 \mathrm{~nm}$ showing an approximately fivefold enhancement in comparison to the initial value (measured by AFM [33]). After etching, the sample is cleaned and mounted [schematically shown in Fig. 1(c)] in a homebuilt confocal setup capable of pulsed optical and microwave pulses (as explained previously in (a)

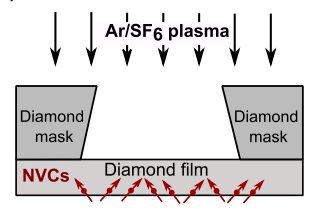

(c)

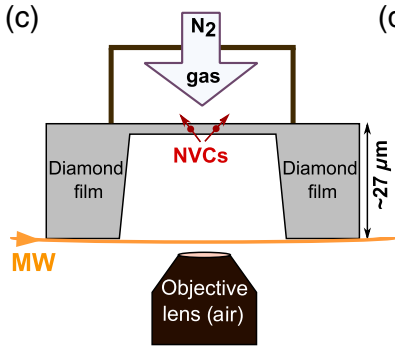

(b)

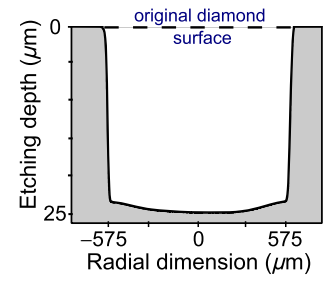

(d)

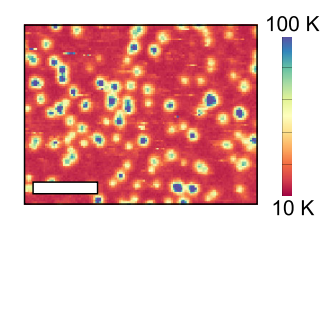

FIG. 1. Fabrication and mounting scheme of the membrane structure. (a) Etching scheme using an $\mathrm{Ar} / \mathrm{SF}_{6}$ plasma-gas mixture and an auxiliary diamond etching mask with an angled-wall hole is illustrated. (b) Depth profile of the diamond film including a thin membrane at the center; an etching depth $>25 \mu \mathrm{m}$ is measured with a Dektak instrument. The slightly thicker part at the rim is due to inhomogeneous etching of the film close to the diamond wall. (c) Schematic of the sample and its mounting in the homebuilt confocal setup for dc measurements under applied pressure. (d) Confocal image of the NVCs near the center of the membrane. The scale bar shows $3 \mu \mathrm{m}$.
Ref. [34]). Figure 1(d) shows a confocal image from the implanted NVCs near the center of the membrane. More detailed information about the fabrication process is given in Appendix B.

\section{MEASUREMENTS IN THE dc REGIME}

\section{A. Optical measurements}

As a first step in obtaining information about the mechanical properties of the membrane, we perform optical measurements of the membrane deflection under a dc pressure through the NVCs as the embedded local nanosensors. To that aim, the sample is installed under a nitrogen-gas-pressure vessel [as depicted schematically in Fig. 1(c)], positioned on top of the confocal setup. The fluorescence PSF ( $z$ axis) of single NVCs near the center of the membrane is monitored as the nitrogen-gas pressure is increased. The applied pressure vs the deflection of the membrane at its center is shown in Fig. 2(a). The obvious nonlinear (cubic polynomial) behavior clearly shows that the final structure thoroughly obeys the CMT of thin circular membranes [Eq. (1)]. Fitting the data using the model [Eq. (1)] yields values of $t=1.2 \pm 0.2 \mu \mathrm{m}$ and $\sigma_{0}=54 \pm 6 \mathrm{MPa}$ for the effective thickness and the radial compressive residual stress, respectively. This derived thickness is in good agreement with the value of approximately 1 to $2 \mu \mathrm{m}$ that is obtained by direct microscopy. As a complementary attempt to assess the sensitivity of the presented hybrid device to pressure, we apply a small pressure while monitoring the fluorescence photon-count rate of a single NVC at the center of the membrane. As can
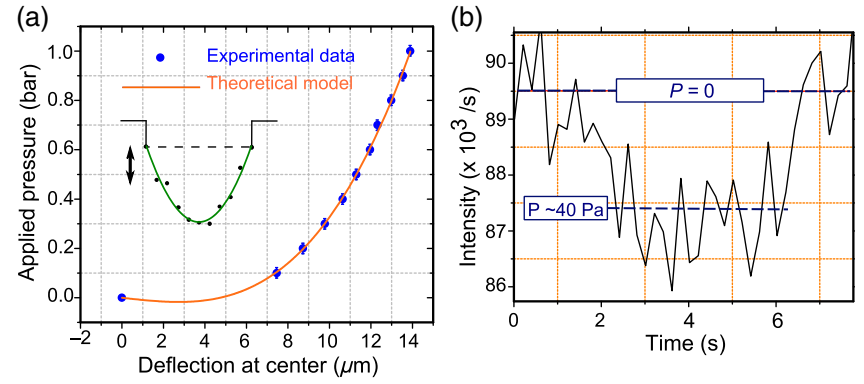

FIG. 2. Optical investigations of the membrane-NVC hybrid system are depicted. (a) Applied dc pressure to the system vs deflection at its center is plotted. The cubic polynomial nature of the curve is a clear characteristic of the mechanics of a thin circular membrane. Vertical error bars demonstrate 0.05 bar, while the horizontal error bars are negligible (nanometer scale). (Inset) Schematic drawing of the membrane, including measurement points and a parabolic fit under static pressure of 0.8 bar in different radial coordinates. Vertical scale bar shows approximately $5 \mu \mathrm{m}$. (b) Recording the fluorescence rate of a single NVC at the center of the membrane in the absence and the presence of a small applied pressure. As explained in the text, this pressure is calculated to be approximately $40 \mathrm{~Pa}$. 
be seen in Fig. 2(b), an approximately $2 \times 10^{3} / \mathrm{s}$ decrease in the photon-count rate is clearly observed. This change in the fluorescence rate can be then attributed to the displacement in $z$ focus of the PSF of the NVC and then converted into an applied dc pressure as small as approximately $40 \mathrm{~Pa}$. In this case, the photon shot-noise limit on the pressure sensitivity is $<6(\mathrm{~Pa} / \sqrt{\mathrm{Hz}})$, which can be improved by increasing the photon-count rate of the NVCs as nanoprobes through incorporating an ensemble of NVCs into this structure. To compare the minimum detected pressure (MDP) of our hybrid device using quantum nanosensors (approximately $40 \mathrm{~Pa}$ ) with recently presented microelectromechanical systems (MEMS)-based pressure sensors, we could say that our device is comparable to the MEMS piezoelectric or capacitive pressure sensors $(\mathrm{MDP} \approx 10-1000 \mathrm{~Pa}$ ) [35-38], but, however, less sensitive in comparison to the flexible sensors, e.g., presented in Ref. [39] $(\mathrm{MDP}=0.1 \mathrm{~Pa})$. See Appendix $\mathrm{C}$ for more details.

\section{B. ODMR measurements}

In the next step, we employ the ground-state spins of the NVCs as nanoprobes of the dc mechanical motion of the membrane. Based on Eq. (6), two different frequency shifts $\left(\Delta_{ \pm}\right)$are expected for two different sets of NVC crystallographic orientations $(A, C$ and $B, D)$ under the (100) diamond surface. However, both shift distributions have identical maxima at the center of the membrane $(r=0)$ [see Fig. 3(a) and 3(b)]. Assuming a thickness and a radius of the membrane equal to 1.2 and $575 \mu \mathrm{m}$, respectively, the longitudinal frequency shift of a single NVC at the center of the membrane is calculated to be

$$
\Delta_{+, \text {center }}=\Delta_{-, \text {center }} \approx 0.012\left(\mathrm{MHz} / \mu \mathrm{m}^{2}\right) \times w^{2},
$$

where $w$ is in microns. To measure this frequency shift, optically detected magnetic-resonance (ODMR) measurements [6] are performed on single NVCs near the center of the membrane. To be confined to the longitudinal frequency shift and to suppress the transverse one, approximately $90 \mathrm{G}$ of magnetic field is aligned to the axis of the NVC under investigation. As plotted in Fig. 3(c), the linear behavior of the longitudinal frequency shift (plotted in absolute values) vs the applied pressure is measured, with a gradient of approximately $2.3 \mathrm{MHz} /$ bar. As shown in Fig. 3(c), an appropriate consistency between the experimental and theoretical curves [based on Eq. (7)] can be seen, which supports our model of the spin-mechanical coupling and mechanics of the membrane. A slight deviation between these two curves can be attributed to the small inhomogeneity in the thickness of the membrane close to its rim as well as to nonideal clamping conditions.

In addition to the applied static pressure, single NVCs are used as local probes to sense the residual stress in the device. Residual stress is an inevitable component of
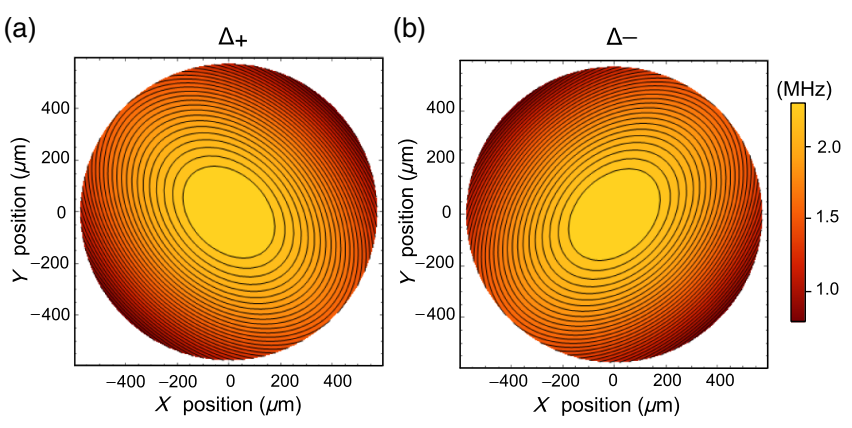

(c)

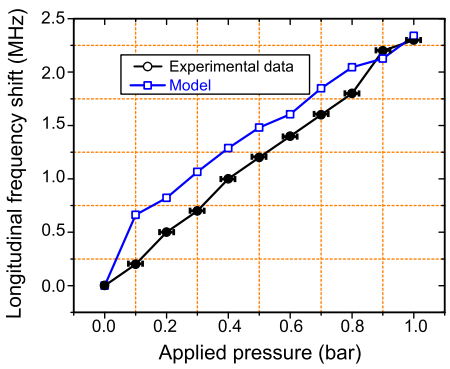

FIG. 3. (a),(b) The simulation of the longitudinal frequency shift (absolute value) distribution over the membrane area is plotted (for an applied pressure of 1 bar) for two different sets of NVC crystallographic orientations. There is an azimuthal asymmetry in both of the plots, which is imposed by a breaking of the symmetry due to the nonsymmetric orientation of the NVCs. (c) The absolute value of the experimental and the model data of the longitudinal frequency shift detected by a single NVC at the center of the membrane is depicted. Our model considering the membrane mechanics and the spin-mechanical interactions predicts similar behavior as the ODMR measurements. The horizontal scale bars demonstrate 0.05 bar.

micro- and nanostructures which causes their mechanical properties to deviate from the expected values [18] and thus complicates and limits their application. As assumed in CMT, the main residual stress in the circular membrane geometry is the radial residual stress due to clamping [18]. To measure this residual stress, we inspect 15 NVCs for each four different orientations located radially at the center of the membrane, and we record the longitudinal frequency shift from the default value of $D=2.870 \mathrm{GHz}$. The average shift is measured to be approximately $0.82 \mathrm{MHz}$. To exclude the residual stress due to the vicinity of the NVCs to the diamond surface, we inspect 30 NVCs implanted in the same depth in a reference sample and, by the same method, obtain the value of approximately $0.57 \mathrm{MHz}$, thus giving a net frequency shift of approximately 0.25 MHz. Based on the spin-stress model and CMT, the residual longitudinal frequency shift at the center of the membrane is equal to $A_{1} \sigma_{0}$. In this way, radial residual stress of $\sigma_{0} \approx 52 \mathrm{MPa}$ is obtained, showing good agreement with $\sigma_{0} \approx 54 \mathrm{MPa}$ achieved through optical measurements. This result highlights the capability of NVCs as sensitive nanoscale probes to characterize the residual stress in diamond mechanical structures. 


\section{MEASUREMENTS IN THE ac REGIME}

Towards implementation of proposals based on NVC spin-oscillator systems $[3,4]$, we study the ac mechanical behavior of the NVC-membrane device. We focus on the lowest order (fundamental) vibrational mode of the membrane that resembles a drum-shaped vibration with maximum amplitude at its center [18]. To excite the fundamental mode, the membrane is installed on top of a piezoelectric chip connected to a frequency generator.

\section{A. Optical measurements}

To acquire the resonance frequency, the fluorescence photon count rate of a single NVC near the center of the membrane is monitored while the frequency of the piezoelectric chip is swept. A dip in the fluorescence intensity at $\omega \approx 2 \pi \times 63 \mathrm{kHz}$ is detected, indicating the fundamental resonance mode of the membrane. The same result is

(a)

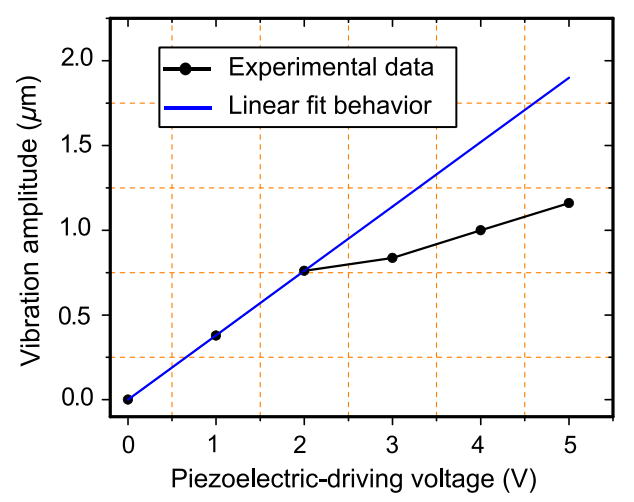

(c)

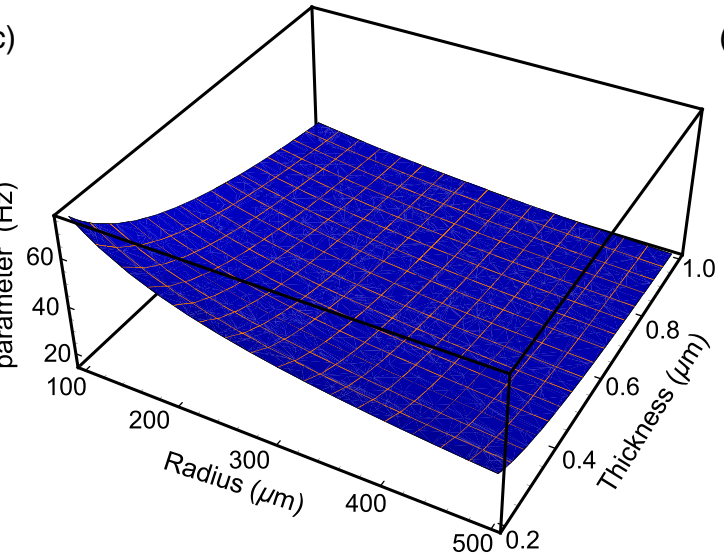

observed using an interferometry method $[12,13,40]$. In both methods, other technical errors, such as the resonance frequency of the piezoelectric chip, are excluded by monitoring the thick part of the diamond film lying far from the membrane. A two-digit quality factor is obtained using both techniques. Since the quality factor is likely to be limited by atmospheric damping, we expect that higher quality factors can be achieved in vacuum [13]. By knowing the fundamental mode frequency of the membrane [18] given by

$$
\omega \cong \frac{2.6}{r \sqrt{\rho}} \sqrt{\sigma_{0}+\frac{4 E t^{2}}{3}}
$$

and its effective mass as $m_{\text {eff }}=1.8 m_{\text {real }}$ [41] where $m_{\text {real }}$ is the real mass of the membrane, the zero-point fluctuation of the membrane [4] will be given by $z_{\mathrm{ZPF}}=$ $\sqrt{\hbar / 2 m_{\mathrm{eff}} \omega} \approx 10^{-16} \mathrm{~m}$.
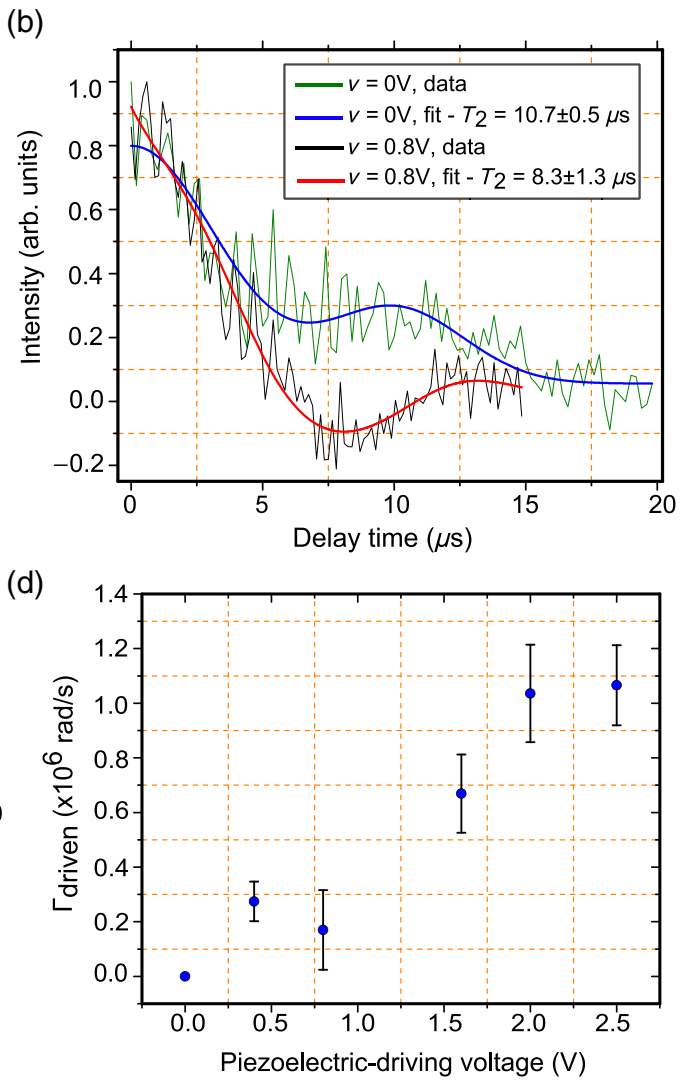

FIG. 4. Optical and Hahn echo measurements in the membrane ac regime. (a) In-resonance vibration amplitude of the membrane vs the piezoelectric-driving voltage is estimated by means of single NVCs, revealing an overall nonlinear behavior. Nevertheless, this behavior can be approximated to a linear fit with slope of $0.38 \mu \mathrm{m} / \mathrm{V}$ for voltages $<2 \mathrm{~V}$. (b) Hahn echo signal under approximately $290 \mathrm{G}$ in the absence (green curve with blue fit [43]) and the presence (black curve with red fit) of in-resonance mechanical driving is demonstrated. An oscillation behavior obeying the Bessel function proves that our system is in the driven regime, as presented theoretically previously [4]. (c) Spin-phonon coupling parameter ( $\lambda$ in Hertz) is shown vs the radius and the thickness of the membrane. In this simulation, the residual stress of the membrane and the magnetic-field gradient are considered to be $100 \mathrm{MPa}$ and $5 \times 10^{6} \mathrm{~T} / \mathrm{m}$, respectively. (d) Driven decoherence rate is plotted vs the driving voltage of the piezoelectric chip. Nonlinear behavior of the membrane oscillator and possible heating effects can cause an ascending decoherence rate. Horizontal error bars in Figs. 4(a) and 4(d) are negligible. 
In the resonant driving regime, the vibrational amplitude of the membrane is estimated optically via an analogous technique to the dc optical measurements. In this way, the vibrationally induced broadening of the PSF [27] of single NVCs near the center of the membrane is estimated vs different piezoelectric-driving voltages [42] [Fig. 4(a)]. Like the displacement with dc pressure, the vibrational amplitude is nonlinear in the piezoelectric-driving voltage. However, for small voltages (less than $2 \mathrm{~V}$ ), it can be seen that the amplitude is approximately linear. In this voltage regime, a linear slope of approximately $0.38 \mu \mathrm{m} / \mathrm{V}$ can be fitted.

\section{B. Spin-echo measurements}

To monitor the coupling of the spin of NVCs to the fundamental mode of the membrane, we perform spin-echo (Hahn echo sequence) measurements on single NVCs near the center of the membrane while its fundamental mode is driven. A magnetic field of approximately $290 \mathrm{G}$ is aligned to the axis of the NVCs. NVCs near the center of the membrane couple dominantly to its fundamental vibration mode. This vibration induces a modulation of the applied magnetic field sensed by the NVC-and thus its spinresonance frequencies $[4,16]$. One can approximate this vibrationally induced interaction between the NVC spin and the membrane oscillator in the linear regime (low piezoelectric voltages) through a periodic detuning term $\lambda \widetilde{z(v)} \cos \omega t$, where $\lambda$ is the coupling parameter of the spin to a membrane vibration quantum, and $\widetilde{z(v)}$ is the voltagedependent dimensionless amplitude of the oscillation (i.e., the vibration amplitude at each piezoelectric-driving voltage divided by $\left.z_{\mathrm{ZPF}}\right)$ [4]. As shown by Bennett et al. [4], this nonsynchronized vibrationally induced detuning results in decoherence of the NVC spin, observable, e.g., in the Hahn echo signal. In this way, the Hahn echo signal depends upon the zeroth-order Bessel function $J_{0}\left[4(\lambda \widetilde{z(v)} / \omega) \sin ^{2}(\omega t / 2)\right]$ (see Appendix D for more details). We observe experimentally [Fig. 4(b)] that under a driving of the membrane at its fundamental vibrational mode, the standard Hahn echo signal shows an oscillation which fits to the Bessel function mentioned above. Using this fit, the coupling parameter is estimated to be $\lambda=6.12 \pm 0.51 \times 10^{-4} \mathrm{rad} / \mathrm{s}$, which corresponds to a vibrationally induced magnetic-field gradient of approximately $27 \mathrm{~T} / \mathrm{m}$. Regarding further realizations of proposals towards quantum-information processing using NVC-diamond mechanical structure hybrid devices [3], the coupling parameter can be drastically improved by reducing the membrane dimension and applying a higher magnetic-field gradient [see Fig. 4(c)]. For instance, by further reducing the radius and the thickness of the membrane to 100 and $0.3 \mu \mathrm{m}$, respectively, and by employing magnetic structures $[44,45]$ introducing magnetic-field gradients of $5 \times 10^{6} \mathrm{~T} / \mathrm{m}$, the coupling parameter $\lambda$ can be improved by 5 orders of magnitude, exceeding $50 \mathrm{~Hz}$. This term plays an important role, while the requirement for a single phonon of the membrane fluctuation to strongly influence the spin coherence of the embedded NVC is evaluated by the cooperativity term, which scales with $\lambda^{2}$ [4].

The resulting driven decoherence rate $\left(\Gamma_{\text {driven }}=\right.$ $2 \pi / T_{2}^{\text {driven }}$ [15] vs piezoelectric-driving voltage is depicted in Fig. 4(d). As shown, it presents an ascending behavior as piezoelectric-driving voltage increases. The precise origin of this voltage-dependent decoherence is not clear. However, we suspect it to be related to the nonlinearity of the membrane's mechanics and related factors, such as the imperfect damping and coupled excitation of multiple vibrational modes. Further investigations would be beyond the scope of this work.

\section{CONCLUSION}

In conclusion, we demonstrate a versatile hybrid system based on a thin circular single-crystal diamond membrane and embedded single NVCs. A certain etching technique is key to producing this hybrid device. Within this architecture, NVCs are used as nanoscale probes to detect the membrane motion in dc and ac regimes, as well as the residual stress of the structure. Theoretical calculations are also provided which support the achieved experimental observations via NVC nanoprobes. The presented results support the potential of diamond quantum hybrid systems to achieve high sensitivity and spatial resolution in piezometry and vibrometry applications. Moreover, such a hybrid system can be exploited in optomechanical cavities [46] and can serve as a platform for photonic structures [47] utilizing NVCs. This work is confined within the groundstate fine structure of single NVCs; however, the excitedstate fine structure offers further opportunities $[48,49]$. One can benefit from the controllable stress in such a system to manipulate the excited-state fine structure of NVCs [50], which is the backbone of their application in quantuminformation processing at low temperature [51-53].

\section{ACKNOWLEDGMENTS}

We acknowledge Thomas Reindl, Thomas Wolf, Thomas Häberle, Roland Nagy, Monika Ubl, Roman Kolesov, Nikolas Abt, Torsten Rendler, and Matthias Widmann for the fruitful discussions. This work is supported by the EU via DIADEMS and SQUTEC, the DFG via FOR 1493 and SPP 1601, and the ARC (Project No. DP140103862). F. F. appreciates the financial support from CNPq Project No. 204246/2013-0.

\section{APPENDIX A: COORDINATE SYSTEM CONVERSION OF STRESS COMPONENTS}

The strain components in the cylindrical coordinate system given in Eq. (2) can be converted to Cartesian system $(x, y)$ in this way: 


$$
\begin{aligned}
\sigma_{x x} & =E\left[\epsilon_{R R} \cos ^{2}(\theta)+\epsilon_{T T} \sin ^{2}(\theta)\right], \\
\sigma_{y y} & =E\left[\epsilon_{R R} \sin ^{2}(\theta)+\epsilon_{T T} \cos ^{2}(\theta)\right], \\
\sigma_{x y} & =E\left(\frac{1}{2} \sin (2 \theta)\left(\epsilon_{R R}-\epsilon_{T T}\right)\right) .
\end{aligned}
$$

\section{APPENDIX B: FABRICATION PROCEDURE}

To realize the planned structure, we start with a CVD electronic grade ${ }^{13} \mathrm{C}$ natural abundance diamond thin film commercially available from Element Six, machined by DDK to a thickness of approximately $27 \mu \mathrm{m}$, with a surface roughness of approximately $2 \mathrm{~nm}$ measured by AFM over several areas of $0.5 \times 0.5 \mu \mathrm{m}$. After cleaning with an aqua regia solution and triacid mixture, we implant nitrogen ions. After cleaning with piranha solution, the sample is heated up to approximately $900^{\circ} \mathrm{C}$ for approximately two hours under a vacuum of $<10^{-6} \mathrm{mbar}$, which is then followed by boiling in a triacid mixture [34] that finally activates single-detectable NVCs. Then we flip the sample, glue it by PMMA on top of a diamond substrate for structural support, and etch the center of it by means of the reactive ion etching-inductively coupled plasma (RIE-ICP) technique based on an $\mathrm{Ar} / \mathrm{SF}_{6}$ gas mixture for $>25 \mu \mathrm{m}$.

The mask is a type-I diamond in which a hole is laser cut at its center by Medidia $\mathrm{GmbH}$ with roughly the same diameter as the final membrane-however, importantly, with angled walls. The etching rate of diamond in the vicinity of the diamond wall is larger than in other places, resulting in the presence of thinner areas close to the rim of the circular membrane which cause cracks in the final structure. By using an angled-wall diamond mask, we can overcome this problem. This task is achieved through the faster corrosion rate of the mask close to the hole in comparison to other places. Therefore, at the rim of the membrane, diamond film is etched less than at its center.

The etching process is done in a Oxford PlasmaPro NGP80 RIE-ICP machine under a vacuum of approximately $10^{-6}$ mbar, a plate temperature of $20^{\circ} \mathrm{C}$, RIE power of $100 \mathrm{~W}$, ICP power of $600 \mathrm{~W}$, and a gas flow of 40 and $25 \mathrm{sccm}$ for $\mathrm{SF}_{6}$ and Ar, respectively, under the chamber pressure of 20 mTorr. After etching, to remove the fluorine termination, we etch the surface for a few nanometers by means of the soft ICP oxygen-plasma technique [32]. The final resulting etching rate is measured by a Dektak instrument to be approximately $170 \mathrm{~nm} / \mathrm{min}$. The resulting surface roughness is measured over a $(1 \times 1)-\mu \mathrm{m}$ area in several positions of the membrane by means of AFM, revealed to be approximately $0.4 \mathrm{~nm}$. This measurement shows an approximately fivefold enhancement of the surface roughness by the presented etching recipe, even after etching more than $25 \mu \mathrm{m}$ of diamond.
APPENDIX C: PRESSURE MEASUREMENTS

The range of pressures over which our device can measure is limited on the (i) low-pressure end by the sensitivity and on the (ii) high-pressure end by the strength of the membrane, as follows.

(i) For a given membrane geometry and location of NVC, the sensitivity is limited by the NVC spincoherence times and the rate of photons collected from the NVC. Therefore, to be able to sense lower pressure, one needs to employ a higher number of embedded NVCs and/or NVCs with longer coherence times.

(ii) The high-pressure end can be taken as equal to the burst pressure of the membrane given by [18]

$$
P_{\text {burst }}=4 \frac{t \sigma_{y}}{R} \sqrt{\frac{3}{2}\left(\sigma_{y}-\sigma_{0}\right) \frac{1-\nu}{E}},
$$

in which $\sigma_{y} \cong 90 \mathrm{GPa}, E \cong 1.2 \times 10^{3} \mathrm{GPa}$, and $\nu \cong$ 0.069 are the yield stress [54], Young's modulus, and Poisson's ratio of diamond, respectively, and $t$, $R$, and $\sigma_{0}$ are the thickness, the radius, and the residual stress of the membrane, respectively. Given the values for diamond and geometry of our presented membrane, the burst pressure is approximately 2500 bar. This value sounds more ideal than realistic for our experiment since breaking of the membrane in the installation points likely occurs at much lower pressures. Nevertheless, it should be noted that, during our measurement, we apply up to 3 bar of dc gas pressure to our device and observe no damage. Considering these two detected pressure limits $\left(\cong 40\right.$ and $\left.\cong 10^{5} \mathrm{~Pa}\right)$, a broad pressuremeasurement range of approximately 4 orders of magnitude is achieved by our hybrid device, while higher orders might be still feasible.

\section{APPENDIX D: SPIN-ECHO MEASUREMENTS}

To perform the spin-coherence measurements, we employ a Hahn echo scheme (similar to that presented in Ref. [34]), while the piezoelectric chip is vibrating the membrane continuously at its fundamental resonance frequency in a nonsynchronized way with respect to the Hahn echo sequence. The resulting Hahn echo signal as a function of waiting time $(\tau)$ can be given by the product of the zeroth-order Bessel function and an exponential decay term based on $T_{2}$ time, as follows [4]:

$$
H(\tau)=A J_{0}\left(4 \frac{\lambda \widetilde{z(v)}}{\omega} \sin ^{2}(\omega \tau)\right) e^{-\chi(\tau)} e^{-\left(\tau / T_{2}\right)^{\gamma}},
$$

where $\lambda$ is the coupling parameter of the NVC's spin to a single-vibration quantum of the membrane, $\omega$ is the eigenfrequency of the membrane oscillator $(2 \pi \times 63 \mathrm{kHz})$, 
$\widetilde{z(v)}$ is the voltage-dependent dimensionless vibration amplitude $\left(\Delta z(v) / z_{\mathrm{ZPF}} ; \Delta z(v)\right.$ is the voltage-dependent vibration amplitude [taken from Fig. 4(a)] and $z_{\mathrm{ZPF}}$ is the zero-point fluctuation of the membrane), $\chi(\tau)$ is the dephasing due to thermal motion, which is negligible in the driven regime [4], and $A$ and $\gamma$ are the fitting parameters. Therefore, by fitting the function in Eq. (D1) to the Hahn echo signal at each piezoelectric voltage, the corresponding values of $T_{2}$ and $\lambda$ can be obtained.

[1] Gershon Kurizki, Patrice Bertet, Yuimaru Kubo, Klaus Mølmer, David Petrosyan, Peter Rabl, and Joerg Schmiedmayer, Quantum technologies with hybrid systems, Proc. Natl. Acad. Sci. U.S.A. 112, 3866 (2015).

[2] M. Wallquist, K. Hammerer, P. Rabl, M. Lukin, and P. Zoller, Hybrid quantum devices and quantum engineering, Phys. Scr. T137, 014001 (2009).

[3] S. D. Bennett, N. Y. Yao, J. Otterbach, P. Zoller, P. Rabl, and M. D. Lukin, Phonon-Induced Spin-Spin Interactions in Diamond Nanostructures: Application to Spin Squeezing, Phys. Rev. Lett. 110, 156402 (2013).

[4] S. D. Bennett, S. Kolkowitz, Q. P. Unterreithmeier, P. Rabl, A. C. Bleszynski Jayich, J. G. E. Harris, and M. D. Lukin, Measuring mechanical motion with a single spin, New J. Phys. 14, 125004 (2012).

[5] M. W. Doherty (to be published).

[6] F. Jelezko and J. Wrachtrup, Single defect centres in diamond: A review, Phys. Status Solidi (a) 203, 3207 (2006).

[7] G. Balasubramanian, I. Y. Chen, R. Kolesov, M. Al-Hmoud, J. Tisler, C. Shin, C. Kim, A. Wojcik, P. R. Hemmer, A. Krueger, T. Hanke, A. Leitenstorfer, R. Bratschlitsch, F. Jelezko, and J. Wrachtrup, Nanoscale imaging magnetometry with diamond spins under ambient conditions, Nature (London) 455, 648 (2008).

[8] T. Staudacher, F. Shi, S. Pezzagna, J. Meijer, J. Du, C. A. Meriles, F. Reinhard, and J. Wrachtrup, Nuclear magnetic resonance spectroscopy on a (5-nanometer $)^{3}$ sample volume, Science 339, 561 (2013).

[9] I. Lovchinsky, A. O. Sushkov, E. Urbach, N. P. de Leon, S. Choi, K. De Greve, R. Evans, R. Gertner, E. Bersin, C. Müller, L. McGuinness, F. Jelezko, R. L. Walsworth, H. Park, and M. D. Lukin, Nuclear magnetic resonance detection and spectroscopy of single proteins using quantum logic, Science 351, 836 (2016).

[10] F. Dolde, H. Fedder, M. W. Doherty, T. Nöbauer, F. Rempp, G. Balasubramanian, T. Wolf, F. Reinhard, L. C. L. Hollenberg, F. Jelezko, and J. Wrachtrup, Electric-field sensing using single diamond spins, Nat. Phys. 7, 459 (2011).

[11] V. Kara, Y.-I. Sohn, H. Atikian, V. Yakhot, M. Lonćar, and K. L. Ekinci, Nanofluidics of single-crystal diamond nanomechanical resonators, Nano Lett. 15, 8070 (2015).

[12] Michael J. Burek, Daniel Ramos, Parth Patel, Ian W. Frank, and Marko Lonćar, Nanomechanical resonant structures in single-crystal diamond, Appl. Phys. Lett. 103, 131904 (2013).
[13] Y. Tao, J. M. Boss, B. A. Moores, and C. L. Degen, Singlecrystal diamond nanomechanical resonators with quality factors exceeding one million, Nat. Commun. 5, 3638 (2014).

[14] Srujan Meesala, Young-Ik Sohn, Haig A. Atikian, Samuel Kim, Michael J. Burek, Jennifer T. Choy, and Marko Lonćar, Enhanced Strain Coupling of Nitrogen-Vacancy Spins to Nanoscale Diamond Cantilevers, Phys. Rev. Applied 5, 034010 (2016).

[15] J. Teissier, A. Barfuss, P. Appel, E. Neu, and P. Maletinsky, Strain Coupling of a Nitrogen-Vacancy Center Spin to a Diamond Mechanical Oscillator, Phys. Rev. Lett. 113, 020503 (2014).

[16] P. Ovartchaiyapong, B. A. Myers, K. W. Lee, and A. C. Bleszynski Jayich, Dynamic strain-mediated coupling of a single diamond spin to a mechanical resonator, Nat. Commun. 5, 4429 (2014).

[17] A. Barfuss, J. Teissier, E. Neu, A. Nunnenkamp, and P. Maletinsky, Strong mechanical driving of a single electron spin, Nat. Phys. 11, 820 (2015).

[18] Werner Schomburg, Introduction to Microsystem Design (Springer, New York, 2011).

[19] Markus Aspelmeyer, Tobias J. Kippenberg, and Florian Marquardt, Cavity optomechanics, Rev. Mod. Phys. 86, 1391 (2014).

[20] J. D. Thompson, B. M. Zwickl, A. M. Jayich, F. Marquardt, S. M. Girvin, and J. G. E. Harris, Strong dispersive coupling of a high-finesse cavity to a micromechanical membrane, Nature (London) 452, 72 (2008).

[21] A. M. Jayich, J. C. Sankey, K. Børkje, D. Lee, C. Yang, M. Underwood, L. Childress, A. Petrenko, S. M. Girvin, and J. G. E. Harris, Cryogenic optomechanics with a $\mathrm{Si}_{3} \mathrm{~N}_{4}$ membrane and classical laser noise, New J. Phys. 14, 115018 (2012).

[22] Thin membranes of well-controlled geometries like circular or rectangular ones should be distinguished from diamond thin films used, e.g., for all-diamond scanning-probe fabrication [23].

[23] Patrick Appel, Elke Neu, Marc Ganzhorn, Arne Barfuss, Marietta Batzer, Micha Gratz, Andreas Tschöpe, and Patrick Maletinsky, Fabrication of all diamond scanning probes for nanoscale magnetometry, Rev. Sci. Instrum. 87, 063703 (2016).

[24] D. R. Glenn, K. Lee, H. Park, R. Weissleder, A. Yacoby, M. D. Lukin, H. Lee, R. L. Walsworth, and C. B. Connolly, Single-cell magnetic imaging using a quantum diamond microscope, Nat. Methods 12, 736 (2015).

[25] C. R. Jacobs, H. Huang, and R. Y. Kwon, Introduction to Cell Mechanics and Mechanobiology (Garland Science, Hamden, CT, 2012).

[26] Ulrich S. Schwarz and Jérôme R. D. Soiné, Traction force microscopy on soft elastic substrates: A guide to recent computational advances, Biochim. Biophys. Acta, Mol. Cell Res. 1853, 3095 (2015).

[27] L. Novotny and B. Hecht, Principles of Nano-Optics (Cambridge University Press, Cambridge, England, 2012).

[28] E. R. MacQuarrie, T. A. Gosavi, N. R. Jungwirth, S. A. Bhave, and G. D. Fuchs, Mechanical Spin Control of Nitrogen-Vacancy Centers in Diamond, Phys. Rev. Lett. 111, 227602 (2013). 
[29] E. R. MacQuarrie, T. A. Gosavi, A. M. Moehle, N. R. Jungwirth, S. A. Bhave, and G. D. Fuchs, Coherent control of a nitrogen-vacancy center spin ensemble with a diamond mechanical resonator, Optica 2, 233 (2015).

[30] M. W. Doherty, J. Michl, F. Dolde, I. Jakobi, P. Neumann, N. B. Manson, and J. Wrachtrup, Measuring the defect structure orientation of a single $\mathrm{NV}^{-}$centre in diamond, New J. Phys. 16, 063067 (2014).

[31] Andrew N. Cleland, Foundations of Nanomechanics (Springer, New York, 2001).

[32] Felipe Fávaro de Oliveira, S. Ali Momenzadeh, Ya Wang, Mitsuharu Konuma, Matthew Markham, Andrew M. Edmonds, Andrej Denisenko, and Jörg Wrachtrup, Effect of low-damage inductively coupled plasma on shallow nitrogen-vacancy centers in diamond, Appl. Phys. Lett. 107, 073107 (2015).

[33] I. Horcas, R. Fernández, J. M. Gómez-Rodríguez, J. Colchero, J. Gómez-Herrero, and A. M. Baro, wsXM: A software for scanning probe microscopy and a tool for nanotechnology, Rev. Sci. Instrum. 78, 013705 (2007).

[34] S. Ali Momenzadeh, Rainer J. Stöhr, Felipe Fávaro de Oliveira, Andreas Brunner, Andrej Denisenko, Sen Yang, Friedemann Reinhard, and Jörg Wrachtrup, Nanoengineered diamond waveguide as a robust bright platform for nanomagnetometry using shallow nitrogen vacancy centers, Nano Lett. 15, 165 (2015).

[35] Yaping Zang, Fengjiao Zhang, Chong-an Di, and Daoben Zhu, Advances of flexible pressure sensors toward artificial intelligence and health care applications, Mater. Horiz. 2, 140 (2015).

[36] Qiugu Wang, Wei Hong, and Liang Dong, Graphene "microdrums" on a freestanding perforated thin membrane for high sensitivity mems pressure sensors, Nanoscale 8 , 7663 (2016).

[37] Wook Choi, Junwoo Lee, Yong Kyoung Yoo, Sungchul Kang, Jinseok Kim, and Jeong Hoon Lee, Enhanced sensitivity of piezoelectric pressure sensor with microstructured polydimethylsiloxane layer, Appl. Phys. Lett. 104, 123701 (2014).

[38] Y. Zhang, R. Howver, B. Gogoi, and N. Yazdi, in Proceedings of the 16th International Conference on Solid-State Sensors, Actuators and Microsystems, Beijing, 2011 (IEEE, New York, 2011), p. 112.

[39] L. Persano, C. Dagdeviren, Y. Su, Y. Zhang, S. Girardo, D. Pisignano, Y. Huang, and J. A. Rogers, High performance piezoelectric devices based on aligned arrays of nanofibers of poly(vinylidenefluoride-co-trifluoroethylene), Nat. Commun. 4, 1633 (2013).

[40] P. Ovartchaiyapong, L. M. A. Pascal, B. A. Myers, P. Lauria, and A.C. Bleszynski Jayich, High quality factor singlecrystal diamond mechanical resonators, Appl. Phys. Lett. 101, 163505 (2012).

[41] I. O. Wygant, M. Kupnik, and B. T. Khuri-Yakub, Analytically calculating membrane displacement and the equivalent circuit model of a circular CMUT cell, in Proceedings of the 2008 IEEE Ultrasonics Symposium, Beijing, 2008, edited by Kendall R. Waters (IEEE, New York, 2008), p. 2111.

[42] L. M. de Lépinay, B. Pigeau, S. Rohr, A. Gloppe, A. Kuhn, P. Verlot, E. Dupont-Ferrier, B. Besga, and O. Arcizet, Nano-optomechanical measurement in the photon counting regime, arXiv: 1503.03200.

[43] B. K. Ofori-Okai, S. Pezzagna, K. Chang, M. Loretz, R. Schirhagl, Y. Tao, B. A. Moores, K. Groot-Berning, J. Meijer, and C. L. Degen, Spin properties of very shallow nitrogen vacancy defects in diamond, Phys. Rev. B 86, 081406 (2012).

[44] Jonilyn G. Longenecker, H. J. Mamin, Alexander W. Senko, Lei Chen, Charles T. Rettner, Daniel Rugar, and John A. Marohn, High-gradient nanomagnets on cantilevers for sensitive detection of nuclear magnetic resonance, ACS Nano 6, 9637 (2012).

[45] I. Jakobi, P. Neumann, Y. Wang, D. Dasari, F. E. Hallak, M. A. Bashir, M. Markham, A. Edmonds, D. Twitchen, and J. Wrachtrup, arXiv:1602.02948.

[46] Erika Janitz, Maximilian Ruf, Mark Dimock, Alexandre Bourassa, Jack Sankey, and Lilian Childress, Fabry-perot microcavity for diamond-based photonics, Phys. Rev. A 92 , 043844 (2015).

[47] Patrik Rath, Sandeep Ummethala, Christoph Nebel, and Wolfram H. P. Pernice, Diamond as a material for monolithically integrated optical and optomechanical devices, Phys. Status Solidi (a) 212, 2385 (2015).

[48] D. Andrew Golter, Thein Oo, Mayra Amezcua, Kevin A. Stewart, and Hailin Wang, Optomechanical Quantum Control of a Nitrogen-Vacancy Center in Diamond, Phys. Rev. Lett. 116, 143602 (2016).

[49] K. W. Lee, D. Lee, P. Ovartchaiyapong, J. Minguzzi, J. R. Maze, and A.C. Bleszynski Jayich, Strain coupling of a mechanical resonator to a single quantum emitter in diamond, arXiv:1603.07680.

[50] A. Batalov, V. Jacques, F. Kaiser, P. Siyushev, P. Neumann, L. J. Rogers, R. L. McMurtrie, N. B. Manson, F. Jelezko, and J. Wrachtrup, Low Temperature Studies of the Excited-State Structure of Negatively Charged Nitrogen-Vacancy Color Centers in Diamond, Phys. Rev. Lett. 102, 195506 (2009).

[51] L. Robledo, L. Childress, H. Bernien, B. Hensen, P. A. Alkemade, and R. Hanson, High-fidelity projective read-out of a solid-state spin quantum register, Nature (London) 477, 574 (2011).

[52] D. D. Bhaktavatsala Rao, Sen Yang, and Jörg Wrachtrup, Generation of entangled photon strings using NV centers in diamond, Phys. Rev. B 92, 081301(R) (2015).

[53] Sen Yang, Ya Wang, Rao D. D. Bhaktavatsala, Thai Hien Tran, S. Ali Momenzadeh, M. Markham, D. J. Twitchen, Ping Wang, Wen Yang, Rainer Stöhr, Philipp Neumann, Hideo Kosaka, and Jörg Wrachtrup, High-fidelity transfer and storage of photon states in a single nuclear spin, Nat. Photonics 10, 507 (2016).

[54] Peter Hess, The mechanical properties of various chemical vapor deposition diamond structures compared to the ideal single crystal, J. Appl. Phys. 111, 051101 (2012). 